\title{
Promoting Cell Growth And Poly-Y-Glutamic Acid Production By Boosting The Synthesis of Alanine And D-Alanyl-D-Alanine In Bacillus Licheniformis
}

\section{Zheng Zhang}

Hubei University

Penghui He

Hubei University

Shiying Hu

Hubei University

Yanqing Yu

Hubei University

Xiaoting Wang

Hubei University

Shouwen Chen ( $\nabla$ mel212@126.com )

Hubei University https://orcid.org/0000-0003-3503-4561

\section{Research Article}

Keywords: Bacillus licheniformis, Alanine and D-alanyl-D-alanine synthesis, Cell growth, Poly-Y-glutamic acid, Metabolic engineering

Posted Date: January 14th, 2022

DOI: https://doi.org/10.21203/rs.3.rs-1234457/v1

License: (c) (1) This work is licensed under a Creative Commons Attribution 4.0 International License.

Read Full License 
1 Promoting cell growth and poly- $\gamma$-glutamic acid production by boosting

2 the synthesis of alanine and D-alanyl-D-alanine in Bacillus licheniformis

3 Zheng Zhang ${ }^{1}$, Penghui $\mathrm{He}^{1}$, Shiying $\mathrm{Hu}^{1}$, Yanqing $\mathrm{Yu}^{1}$, Xiaoting Wang ${ }^{1}$, Shouwen $\mathrm{Chen}^{1 *}$

4

$5{ }^{1}$ State Key Laboratory of Biocatalysis and Enzyme Engineering, Environmental Microbial Technology

6 Center of Hubei Province, College of Life Sciences, Hubei University, Wuhan 430062, China

$7 \quad{ }^{*}$ Corresponding author: Prof. Shouwen Chen

8 Tel./fax.: $+86027-88666081$.

$9 \quad$ E-mail address: mel212@126.com (S. Chen).

10

Postal address: 368 Youyi Avenue, Wuchang District, Wuhan 430062, Hubei, PR China 
Objective The production of some bio-chemicals affected by the cell growth. This study aimed at promoting the cell growth by overexpressing the synthesis of peptidoglycans tetrapeptide tail components to improve poly- $\gamma$-glutamic acid ( $\gamma$-PGA) production.

Results L-alanine, D-alanine and D-alanyl-D-alanine are primary precursors for the synthesis of peptidoglycans tetrapeptide tail. The addition of L-alanine and D-alanine significantly increased both the cell growth and production of $\gamma$-PGA. Then, several genes encoding key enzymes for L/D-alanine and D-alanyl-D-alanine biosynthesis were overexpressed respectively, including ald (encoding alanine dehydrogenase), $d a l$ (encoding alanine racemase) and $d d l$ (encoding D-alanine ligase). The results showed that the overexpression of genes ald, $d a l$ and $d d l$ increased the production of $\gamma$-PGA by $19.72 \%$, $15.91 \%$ and $60.90 \%$, and increased the microbial biomass by $15.58 \%, 18.34 \%$ and $49.85 \%$, respectively. Moreover, we demonstrated that the overexpression of genes ald, dal and $d d l$ increased $\gamma$-PGA production mainly by enhancing cell growth rather than providing more precursors. to the cell growth and the high yield of $\gamma$-PGA, and provided an effective strategy for producing $\gamma$-PGA.

\section{Keywords}




\section{Introduction}

Bacterial cell walls are characterized by the presence of peptidoglycans, macromolecules built from sugars and peptides, which help to maintain cell shape and integrity and balance the intracellular osmotic pressure (Brown et al. 2020). Bacterial cell wall synthesis is closely related to cell growth, and disruption of proper bacterial cell wall formation makes the cell highly sensitive to common environmental pressure, such as high salinity or antibiotics (Das et al. 2011). Thus, the methods of engineering cell wall component were generally developed to improve cell integrity and production of biochemicals. For instance, Son et al. suppressed cell lysis and increased squalene production by approximately $12 \%$ through activating the cell wall integrity pathway (Son et al. 2020). In addition, elevation of membrane cardiolipin biosynthesis and repression of the cell division initiator protein FtsZ also increased the $\mathrm{OD}_{600}$ by $86 \%$ and increased the $\mathrm{HA}$ titer by $204 \%$ (Westbrook et al. 2018). Therefore, engineering cell wall component or shape might be feasible strategies to increase metabolites production. L-alanine (L-Ala), D-alanine (D-Ala) and D-alanyl-D-alanine are important components of the tetrapeptide tail in peptidoglycans of bacterial cell wall, which play an indispensable role in the normal growth of bacteria (Fig. 1) (Das et al. 2011). In recent studies, D-alanine was found to be essential for cell growth, biofilm formation and interspecific competition (Qiu et al. 2016), and it was often used as a screening marker for bacterial auxotroph to construct food-grade expression systems (Xia et al. 2007). Poly- $\gamma$-glutamic acid ( $\gamma$-PGA), a multifunctional biopolymer made up of D-glutamic acid and / or L-glutamic acid monomer by $\gamma$-amide bond, was selected as a research objective (Luo et al. 2016). Generally, numerous methods were developed to improve the $\gamma$-PGA production, such as increasing the supply of precursors, blocking the synthesis of by-products, boosting energy supplement, et al (Cai et al. 2018; Feng et al. 2015). While, the high viscosity of $\gamma$-PGA decreased the concentrations of dissolved 
oxygen and limited the absorption or utilization of nutrients during the fermentation, which property hindered the cell growth and the synthesis of $\gamma$-PGA (Hsueh et al. 2017). Previous study in our lab found that the decrease of negative charge on cell wall surface could significantly improve cell growth and $\gamma$ PGA production in B. licheniformis (He et al. 2019). So, cell wall properties are closely related to cell growth and $\gamma$-PGA synthesis. In this study, alanine dehydrogenase (encode by ald), alanine racemase (encode by $d a l$ ), D-alanyl-D-alanine ligase (encode by $d d l$ ) were respectively overexpressed to explore the effects of L/D-alanine and D-alanyl-D-alanine which associated with peptidoglycans tetrapeptide tail synthesis on cell growth and $\gamma$-PGA production.

Fig. 1

\section{Materials and methods}

Strains and plasmids. The strains and plasmids used in this study were listed in Table 1. $B$. licheniformis WX-02 (CCTCC M208065) was served as the original strain for the construction of recombinant strains. The ald, $d a l$ and $d d l$ overexpressed vector pHY-ald, pHY-dal and pHY-ddl were constructed based on shuttle vector pHY300PLK.

\section{Table 1}

Medium and cultivation conditions. LB medium $(10 \mathrm{~g} / \mathrm{L}$ Tryptone, $5 \mathrm{~g} / \mathrm{L}$ yeast extract, $10 \mathrm{~g} / \mathrm{L}$ $\mathrm{NaCl}, \mathrm{pH}$ 7.2) was served as the basic medium for the cultivation of B. licheniformis and E. coli, and 20 $\mu \mathrm{g} / \mathrm{mL}$ kanamycin, $50 \mu \mathrm{g} / \mathrm{mL}$ ampicillin or $20 \mu \mathrm{g} / \mathrm{mL}$ tetracycline were added into the medium when necessary. The seed culture of $B$. licheniformis was prepared in $250 \mathrm{~mL}$ flasks with $50 \mathrm{~mL} \mathrm{LB}$ medium, and incubated in the rotatory shaker with $180 \mathrm{rpm}$ at $37^{\circ} \mathrm{C}$ for $10-12 \mathrm{~h}$ until $\mathrm{OD}_{600}$ reached 4.0 4.5. Then, the seeds were transferred into the $\gamma$-PGA production medium, consisting of (per liter) $80 \mathrm{~g}$ glucose, 10 g sodium citrate, $8 \mathrm{~g} \mathrm{NH}_{4} \mathrm{Cl}, 1 \mathrm{~g} \mathrm{CaCl}, 1 \mathrm{~g} \mathrm{~K}_{2} \mathrm{HPO}_{4} \cdot 3 \mathrm{H}_{2} \mathrm{O}, 1 \mathrm{~g} \mathrm{MgSO} \cdot 7 \mathrm{H}_{2} \mathrm{O}, 1 \mathrm{~g} \mathrm{ZnSO} \cdot 7 \mathrm{H}_{2} \mathrm{O}$ and 0.15 
$\mathrm{g} \mathrm{MnSO}_{4} \cdot 7 \mathrm{H}_{2} \mathrm{O}$ at pH 7.2. The fermentation was performed in the rotatory shaker with $220 \mathrm{rpm}$ at $37^{\circ} \mathrm{C}$ for $36 \mathrm{~h}$.

Construction of the ald, dal and ddl overexpressed strain. The method for constructing the ald overexpression strain was used as an example and which referred to the previous method (Cai et al. 2018). Briefly, P43 promoter from B. subtilis 168, ald gene and amyL terminator from B. licheniformis WX-02 were amplified by the corresponding primers (Table 2), fused by SOE-PCR, and the fused fragment was inserted into pHY300 at the restriction sites $X b a$ I and $E c o$ R I. PCR verification and DNA sequence confirmed that the ald expression vector was constructed successfully, named pHY-ald. Then, pHY-ald was electro-transferred into B. licheniformis WX-02 to construct the ald overexpression strain, named WX-02/pHY-ald. The strain harboring the empty pHY300PLK was used as the control. Engineered strains WX-02/pHY-ald, WX-02/pHY-dal and WX-02/pHY- $d d l$ were constructed with the same method.

\section{Table 2}

\section{Analytical methods}

Cell growth curve was determined by measuring the optical density at $600 \mathrm{~nm}\left(\mathrm{OD}_{600}\right)$ in $\mathrm{LB}$ medium. $\gamma$-PGA titer and biomass were detected by the method described in the previous research $(\mathrm{He}$ et al. 2019). Concentrations of acetoin, 2,3-butanediol and acetic acid were quantified using Thermo Scientific GC-MS (Thermo, USA) equipped with TG-WAXMS column $(30 \mathrm{~m} \times 0.32 \mathrm{~mm}$ ID, $0.25 \mu \mathrm{m}$ film) by an optimized method from previous research (Qi et al. 2014). The method for determination of alanine and glutamic acid concentrations were accorded to the previous reported method (Yuan et al. 2019) by Thermo Scientific GC-MS (Thermo, USA) equipped with TG-5MS column $(30 \mathrm{~m} \times 0.32 \mathrm{~mm}$ ID, $0.25 \mu \mathrm{m}$ film). The values shown represent the means of three independent experiments and the error bars represent standard deviations of three values. 


\section{Results and discussions}

The addition of L-alanine or D-alanine can promote the cell growth and $\gamma$-PGA production.

In order to investigate the effects of L-alanine or D-alanine on cell growth, growth curves of $B$. licheniformis WX-02 were measured upon addition of different concentrations of exogenous Lalanine or D-alanine to the LB media (Fig. 2). Firstly, the $\mathrm{OD}_{600}$ of WX-02 in LB medium with L-alanine addition were detected (Fig. 2A), which showed a significant increasement in cell growth when $0.1 \mathrm{~g} / \mathrm{L}$ L-alanine was added to the medium compared with the control group (no L-alanine addition). It was worthy for us to note that the cell growth does not always improve with the concentration of added Lalanine increases. The $\mathrm{OD}_{600}$ of cells were even decreased when $0.2,0.3$ and $0.4 \mathrm{~g} / \mathrm{L}$ of L-alanine were added to the medium compared with that of $0.1 \mathrm{~g} / \mathrm{L}$. These results suggested that the addition of L-alanine can boost bacterial cell growth, but the concentration of added L-alanine was need to be considered.

Then, the effect of D-alanine on cell growth was explored in WX-02 (Fig. 2B). The addition of Dalanine in medium promoted the cell growth of WX-02, and the $\mathrm{OD}_{600}$ increased most with $0.1 \mathrm{~g} / \mathrm{L} \mathrm{D}-$ alanine addition. From the above results, it was found that an appropriate concentration of L/D-alanine could promote the cell growth, and the high concentration of L/D-alanine could hinder cell growth.

Fig. 2

The effects of L-alanine and D-alanine on $\gamma$-PGA synthesis were also investigated by adding different concentrations of L-alanine and D-alanine into $\gamma$-PGA fermentation medium. The results showed that the supplement of L-alanine and D-alanine could effectively improve $\gamma$-PGA production (Fig. 3). The titer of $\gamma$-PGA was increased by $14.92 \%$ compared with the control (without L-alanine addition) when $0.2 \mathrm{~g} / \mathrm{L}$ L-alanine was added into the medium (Fig. 3A). In addition, the $\gamma$-PGA titer was also respectively increased by $5.89 \%, 6.19 \%$ and $6.04 \%$ when adding $0.1,0.2$ and $0.3 \mathrm{~g} / \mathrm{L}$ of D-alanine 
119 (Fig. 3B). Therefore, the results indicated that the addition of L-alanine and D-alanine with appropriate concentrations was beneficial to the cell growth and further facilitated $\gamma$-PGA production.

Fig. 3

Overexpression of $\boldsymbol{a l d}, \boldsymbol{d a l}$ and $\boldsymbol{d} \boldsymbol{d l}$ promotes $\boldsymbol{\gamma}$-PGA production. L-alanine and D-alanine are

primary precursors for D-alanyl-D-alanine synthesis, and D-alanyl-D-alanine is subsequently involved

in the synthesis of tetrapeptide tails during peptidoglycan assembly (Fig. 1). In order to explore effects of endogenous generation of L-alanine, D-alanine and D-alanyl-D-alanine on the synthesis of $\gamma$-PGA, alanine dehydrogenase (Ald), alanine racemase (Dal) and D-alanine ligase (Ddl) were individually overexpressed in B. licheniformis WX-02 to construct engineered strains WX-02/pHY-ald, WX-02/pHY$d a l$ and WX-02/pHY-ddl, and the strain WX-02/pHY300 which harboring the empty pHY300PLK was used as the control. The results showed that the overexpression of the genes ald, $d a l$ and $d d l$ increased the $\gamma$-PGA titer by $19.72 \%, 15.91 \%$ and $60.90 \%$, respectively (Fig. $4 \mathbf{A}$ ). The biomass of WX-02/pHYald, WX-02/pHY-dal and WX-02/pHY-ddl were also increased by $13.96 \%, 15.58 \%$ and $49.85 \%$, respectively (Fig. 4A). It was worth noting that the $\gamma$-PGA yield of WX-02/pHY-ald, WX-02/pHY-dal and WX-02/pHY-ddl had no significant difference compared with that of the control strain (Fig. 4A), promoting cell growth. overexpression. Compared with the control, the intracellular alanine concentration of WX-02/pHY-ald and WX-02/pHY-dal increased by $272.24 \%$ and $235.95 \%$, respectively, and the concentration of intracellular alanine decreased by $55.12 \%$ in WX-02/pHY- $d d l$ (Fig. 4B). Those results illustrated that the 
141 acid is the direct precursor for $\gamma$-PGA synthesis and the accumulation of glutamic acid affected the $\gamma$ -

142 PGA production (Tian et al. 2017). The accumulation of glutamic acid in ald, dal and ddl overexpressed

143 strains had no significant difference compared with the control strain (Fig. 4B), which further indicated

144 that $\gamma$-PGA synthesis was promoted by enhancing cell growth rather than affecting its precursors

145 supplement when ald, dal and $d d l$ were overexpressed.

Fig. 4

Effects of genes ald, dal and $d d l$ overexpression on by-products synthesis. Acetoin, 2,3-

butanediol, acetic acid and lactic acid are main by-products generated during $\gamma$-PGA synthesis, which

consumed partial carbon fluxes. Many previous studies have shown that the production of the target biochemicals can be increased by reducing the synthesis of by-products (Ma et al. 2018). Meanwhile, the synthesis of by-products requires pyruvate as the precursor, and pyruvate is also the direct precursor for alanine synthesis. Thus, the accumulations of acetoin, 2,3-butanediol, acetic acid and lactic acid were detected in ald, $d a l$ and $d d l$ overexpressed strains. The results showed that the by-products were respectively reduced by $14.10 \%, 8.77 \%$ and $36.62 \%$ when overexpressing gene ald, dal and $d d l$ compared with the control strain (Table 3). It showed us that the overexpression of genes ald, dal and $d d l$ leaded a significant decrease in the synthesis of by-products, which was more conducive for cell growth and $\gamma$-PGA synthesis.

\section{Conclusion} $\gamma$-PGA is a kind of multifunctional biopolymer with many applications, and its high viscosity

161 hinders the oxygen transformation and the nutrients absorption and utilization, which further affects the cell growth and $\gamma$-PGA synthesis. In this study, we confirmed that the synthesis of L/D-alanine and D- 
alanyl-D-alanine can promote the cell growth, and the enhancement of alanine and D-alanyl-D-alanine was an effective approach to improve the $\gamma$-PGA production.

Author contributions SW Chen designed experiments, contributed reagents and materials. Z Zhang, PH He, SY Hu, YQ Yu and XT Wang performed the experiments. Z Zhang and PH He drafted the manuscript.

\section{Acknowledgments}

This work was supported by the National Key Research and Development Program of China (2021YFC2100202)

\section{Declarations}

\section{Conflict of interest}

The authors declare no conflicts of interest.

\section{Ethical approval}

This article does not contain any studies with human participants performed by any of the authors.

\section{References}

Brown AR, Gordon RA, Hyland SN, Siegrist MS, Grimes CL (2020) Chemical Biology Tools for Examining the Bacterial Cell Wall. Cell Chem Biol 27(8):1052-1062 doi:10.1016/j.chembiol.2020.07.024

Cai D, Chen Y, He P, Wang S, Mo F, Li X, Wang Q, Nomura CT, Wen Z, Ma X, Chen S (2018) Enhanced production of poly-gamma-glutamic acid by improving ATP supply in metabolically engineered Bacillus licheniformis. Biotechnol Bioeng 115(10):2541-2553 doi:10.1002/bit.26774

Das D, Herve M, Feuerhelm J, Farr CL, Chiu HJ, Elsliger MA, Knuth MW, Klock HE, Miller MD, 
Godzik A, Lesley SA, Deacon AM, Mengin-Lecreulx D, Wilson IA (2011) Structure and function of the first full-length murein peptide ligase (Mpl) cell wall recycling protein. PLoS One 6(3):e17624 doi:10.1371/journal.pone.0017624

Feng J, Gu Y, Quan Y, Cao M, Gao W, Zhang W, Wang S, Yang C, Song C (2015) Improved poly-gammaglutamic acid production in Bacillus amyloliquefaciens by modular pathway engineering. Metab Eng 32:106-115 doi:10.1016/j.ymben.2015.09.011

He P, Wan N, Cai D, Hu S, Chen Y, Li S, Chen S (2019) ${ }^{13}$ C-Metabolic flux analysis reveals the metabolic flux redistribution for enhanced production of poly-gamma-glutamic acid in dlt over-expressed Bacillus licheniformis. Front Microbiol 10:105 doi:10.3389/fmicb.2019.00105

Hsueh YH, Huang KY, Kunene SC, Lee TY (2017) Poly-gamma-glutamic acid synthesis, gene regulation, phylogenetic relationships, and role in fermentation. Int J Mol Sci 18(12) doi:10.3390/ijms18122644

Luo Z, Guo Y, Liu J, Qiu H, Zhao M, Zou W, Li S (2016) Microbial synthesis of poly-gamma-glutamic acid: current progress, challenges, and future perspectives. Biotechnol Biofuels 9:134 doi:10.1186/s13068-016-0537-7

Ma W, Liu Y, Shin HD, Li J, Chen J, Du G, Liu L (2018) Metabolic engineering of carbon overflow metabolism of Bacillus subtilis for improved N-acetyl-glucosamine production. Bioresour Technol 250:642-649 doi:10.1016/j.biortech.2017.10.007

Qi G, Kang Y, Li L, Xiao A, Zhang S, Wen Z, Xu D, Chen S (2014) Deletion of meso-2,3-butanediol dehydrogenase gene budC for enhanced D-2,3-butanediol production in Bacillus licheniformis. Biotechnol Biofuels 7(1):16 doi:10.1186/1754-6834-7-16 

Oral Microbiol 31(5):435-44 doi:10.1111/omi.12146

Son SH, Kim JE, Oh SS, Lee JY (2020) Engineering Cell Wall Integrity Enables Enhanced Squalene Production in Yeast. J Agric Food Chem 68(17):4922-4929 doi:10.1021/acs.jafc.0c00967

Tian G, Wang Q, Wei X, Ma X, Chen S (2017) Glutamate dehydrogenase (RocG) in Bacillus licheniformis WX-02: Enzymatic properties and specific functions in glutamic acid synthesis for poly-gamma-glutamic acid production. Enzyme Microb Technol 99:9-15 doi:10.1016/j.enzmictec.2017.01.002

Westbrook AW, Ren X, Moo-Young M, Chou CP (2018) Engineering of cell membrane to enhance heterologous production of hyaluronic acid in Bacillus subtilis. Biotechnol Bioeng 115(1):216231 doi:10.1002/bit.26459

Xia Y, Chen W, Zhao J, Tian F, Zhang H, Ding X (2007) Construction of a new food-grade expression system for Bacillus subtilis based on theta replication plasmids and auxotrophic complementation. Appl Microbiol Biotechnol 76(3):643-50 doi:10.1007/s00253-007-1035-4

Yuan H, Xu Y, Chen Y, Zhan Y, Wei X, Li L, Wang D, He P, Li S, Chen S (2019) Metabolomics analysis reveals global acetoin stress response of Bacillus licheniformis. Metabolomics 15(3):25 doi:10.1007/s11306-019-1492-7 
Fig. 1 Graphical representation of alanine synthesis (A) and peptidoglycan assembly (B). PYR: pyruvate; OAA: oxaloacetic acid; CIT: citric acid; ICIT: isocitric acid; AKG: $\alpha$-ketoglutaric acid; SucCoA: succinyl-coenzyme A; SUC: succinic acid; FUM: fumaric acid; MAL: malic acid; AceCoA: acetyl coenzyme A; L-Glu: L-glutamic acid; D-Glu: D- glutamic acid; L-Ala: L-alanine; D-Ala: Dalanine; L-Lys: L-lysine; 2,3-BDO: 2,3- butanediol. Fig. 2 Effects of L/D-alanine addition with different concentrations on the growth curves of $B$. licheniformis WX-02. (A): Adding L-alanine; (B): Adding D-alanine.

Fig. 3 Effects of L/D-alanine addition with different concentrations on cell growth and $\gamma$-PGA production. (A): Adding L-alanine; (B): Adding D-alanine. and glutamic acid were detected in mid-log phase. 
Table 1 The strains and plasmids used in this research

\begin{tabular}{|c|c|c|}
\hline Strains and plasmids & Relevant properties & Source \\
\hline \multicolumn{3}{|l|}{ Strains } \\
\hline Escherichia coli DH5 $\alpha$ & $\begin{array}{l}\text { supE44 } \Delta l a c \mathrm{U} 169 \text { (f } 80 \text { lacZuM15) hsd } \\
\mathrm{R} 17 \text { recA1 gyrA96 thi1 relA1 }\end{array}$ & Stored in this lab \\
\hline Bacillus licheniformis WX-02 & Wide-type CCTCC M208065 & Stored in this lab \\
\hline $\mathrm{WX}-02 / \mathrm{pHY}$-ald & WX-02 harboring $\mathrm{pHY}$-ald & This study \\
\hline WX-02/pHY-dal & WX-02 harboring $\mathrm{pHY}-\mathrm{dal}$ & This study \\
\hline WX-02/pHY- $d d l$ & WX-02 harboring $\mathrm{pHY}-d d l$ & This study \\
\hline \multicolumn{3}{|l|}{ Plasmids } \\
\hline pHY300PLK & E. coli and B. s shuttle vector; $\mathrm{Amp}^{\mathrm{r}}, \mathrm{Tet}^{\mathrm{r}}$ & This study \\
\hline pHY-ald & ald expression vector & This study \\
\hline pHY-dal & dal expression vector & This study \\
\hline $\mathrm{pHY}-d d l$ & $d d l$ expression vector & This study \\
\hline
\end{tabular}


Table 2 PCR primers used in this study

\begin{tabular}{ll}
\hline Primers & Sequence \\
\hline pHY-F & GTTTATTATCCATACCCTTAC \\
pHY-R & CAGATTTCGTGATGCTTGTC \\
P43-F1 & GCGAATTCTGATAGGTGGTATGTTTTCG \\
P43-R1 & CTTTCGGTACGCCGATAATCATGTGTACATTCCTCTCTTACCTA \\
ald-F & TAGGTAAGAGAGGAATGTACACATGATTATCGGCGTACCGAAAG \\
ald-R & GAAATCCGTCCTCTCTGCTCTTCTATGCGCCGGCGGCTGACGAC \\
TamyL-F1 & GTCGTCAGCCGCCGGCGCATAGAAGAGCAGAGAGGACGGATTTC \\
TamyL-R1 & GGTCTAGACGCAATAATGCCGTCGCACT \\
P43-F2 & GCGAATTCTGATAGGTGGTATGTTTTCG \\
P43-R2 & TATAGAATGGTTTTAAGCTCATGTGTACATTCCTCTCTTACCTA \\
$d a l-F$ & TAGGTAAGAGAGGAATGTACACATGAGCTTAAAACCATTCTATA \\
$d a l-R$ & GAAATCCGTCCTCTCTGCTCTTTCAGGATTGATCAGGCAAGATC \\
TamyL-F2 & GATCTTGCCTGATCAATCCTGAAAGAGCAGAGAGGACGGATTTC \\
TamyL-R2 & GGTCTAGACGCAATAATGCCGTCGCACT \\
P43-F3 & GCGAATTCTGATAGGTGGTATGTTTTCG \\
P43-R3 & CCAATCCTAATCTTGTCTTCAAGTGTACATTCCTCTCTTACCTA \\
$d d l-F$ & TAGGTAAGAGAGGAATGTACACTTGAAGACAAGATTAGGATTGG \\
$d d l-R$ & GAAATCCGTCCTCTCTGCTCTTTTAAAATGTATGTTTAATCTG \\
TamyL-F3 & CAGATTAAACATACATTTTAAAAGAGCAGAGAGGACGGATTTC \\
TamyL-R3 & GGTCTAGACGCAATAATGCCGTCGCACT \\
\hline
\end{tabular}


Table 3 Acetoin, 2,3-butanediol, lactate and acetic acid synthesis by engineered strains

\begin{tabular}{cccccc}
\hline Strains & $\begin{array}{c}\text { Acetoin } \\
(\mathrm{g} / \mathrm{L})\end{array}$ & $\begin{array}{c}2,3 \text {-Butanediol } \\
(\mathrm{g} / \mathrm{L})\end{array}$ & $\begin{array}{c}\text { Acetic acid } \\
(\mathrm{g} / \mathrm{L})\end{array}$ & $\begin{array}{c}\text { Lactic acid } \\
(\mathrm{g} / \mathrm{L})\end{array}$ & $\begin{array}{c}\text { By-products } \\
(\mathrm{g} / \mathrm{L})\end{array}$ \\
\hline WX-02/pHY300 & $6.31 \pm 0.17$ & $14.88 \pm 0.72$ & $6.69 \pm 0.14$ & $0.85 \pm 0.03$ & 28.73 \\
WX-02/pHY-ald & $6.82 \pm 0.06$ & $11.65 \pm 0.43$ & $5.74 \pm 0.20$ & $0.48 \pm 0.03$ & 24.68 \\
WX-02/pHY-dal & $9.89 \pm 0.10$ & $10.62 \pm 0.17$ & $5.03 \pm 0.21$ & $0.66 \pm 0.02$ & 26.21 \\
WX-02/pHY-ddl & $5.65 \pm 0.13$ & $7.45 \pm 0.68$ & $4.69 \pm 0.19$ & $0.43 \pm 0.02$ & 18.21 \\
\hline
\end{tabular}

247 


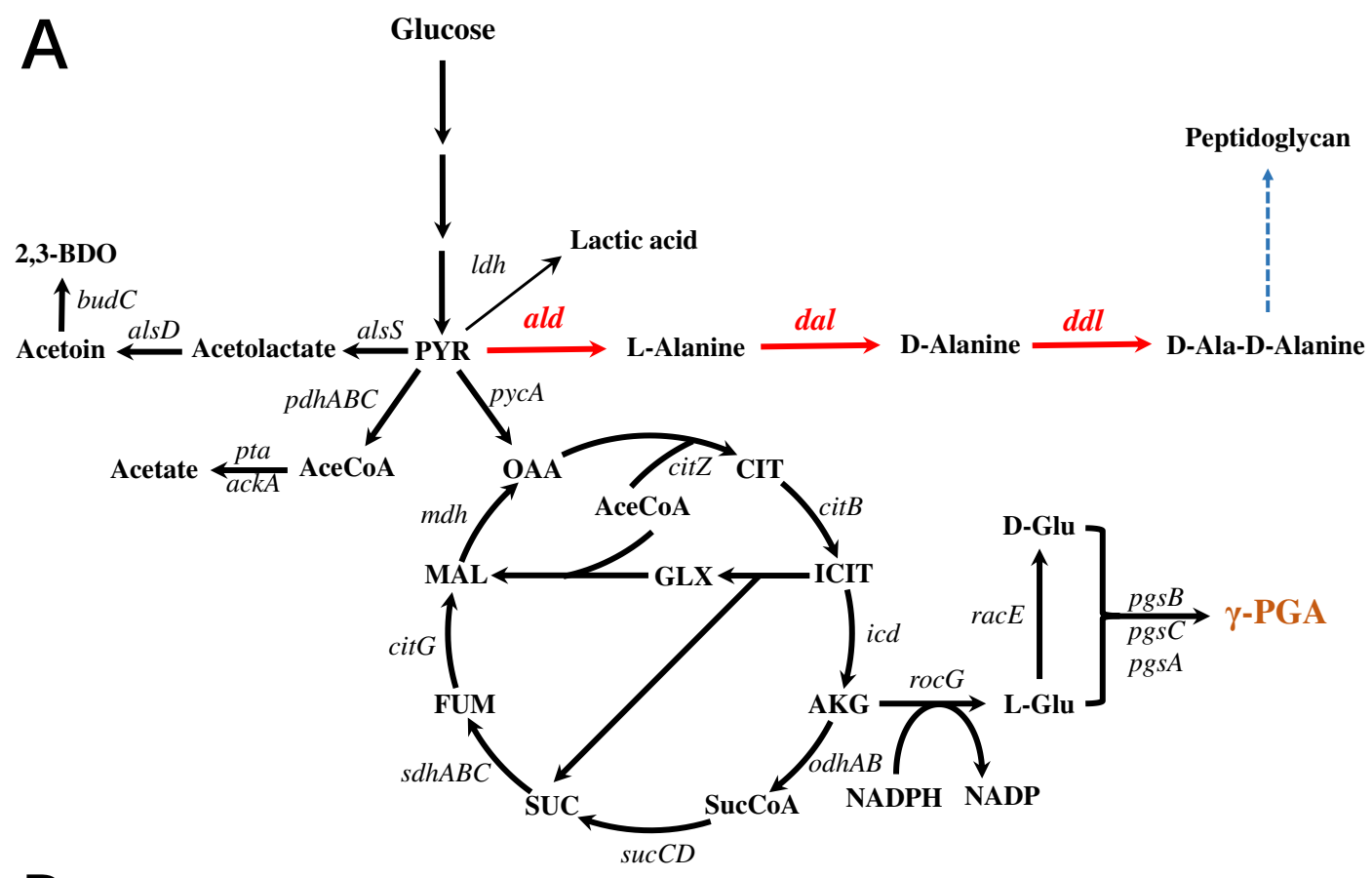

B

UDP-MurNAc-L-Ala-D-Glu-L-lys-D-Ala-D-Ala D-Ala-D-Ala $\uparrow$ mur $F$ UDP-MurNAc-L-Ala-D-Glu-L-lys $\uparrow$ murE UDP-MurNAc-L-Ala-D-Glu

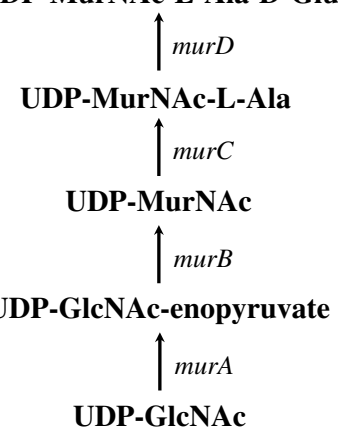
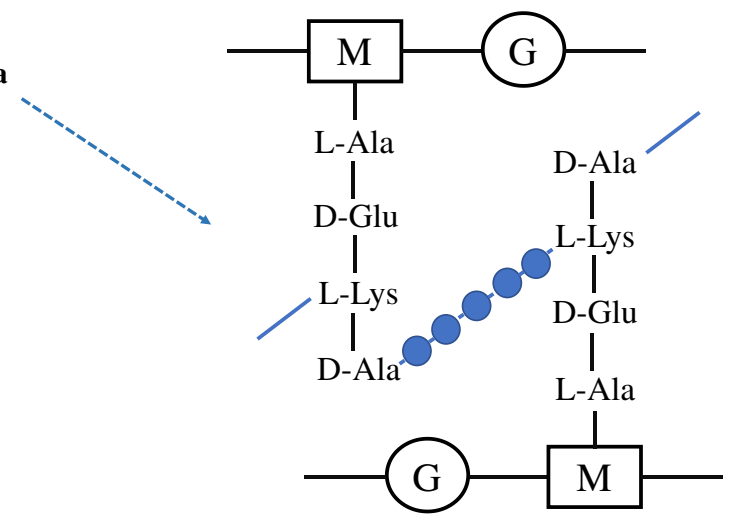

Peptidoglycan

$\mathrm{M}$ N-acetylmuramic acid

G) N-acetylglucosamine

Fig. 1 Graphical representation of alanine synthesis (A) and peptidoglycan assembly (B). PYR: pyruvate; OAA: oxaloacetic acid; CIT: citric acid; ICIT: isocitric acid; AKG: $\alpha$-ketoglutaric acid; SucCoA: succinyl-coenzyme A; SUC: succinic acid; FUM: fumaric acid; MAL: malic acid; AceCoA: acetyl coenzyme A; L-Glu: L-glutamic acid; D-Glu: D- glutamic acid; L-Ala: L-alanine; D-Ala: D- 

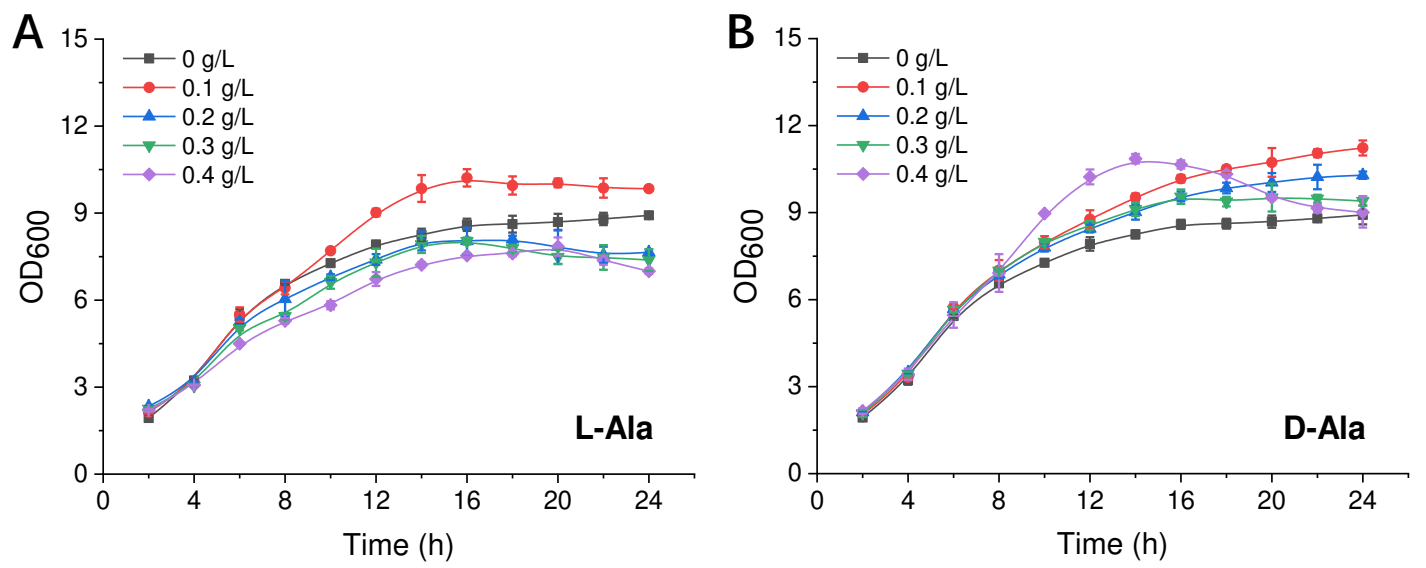

256 Fig. 2 Effects of $\mathbf{L} / \mathbf{D}$-alanine addition with different concentrations on the growth curves of $B$.

licheniformis WX-02. (A): Adding L-alanine; (B): Adding D-alanine. 

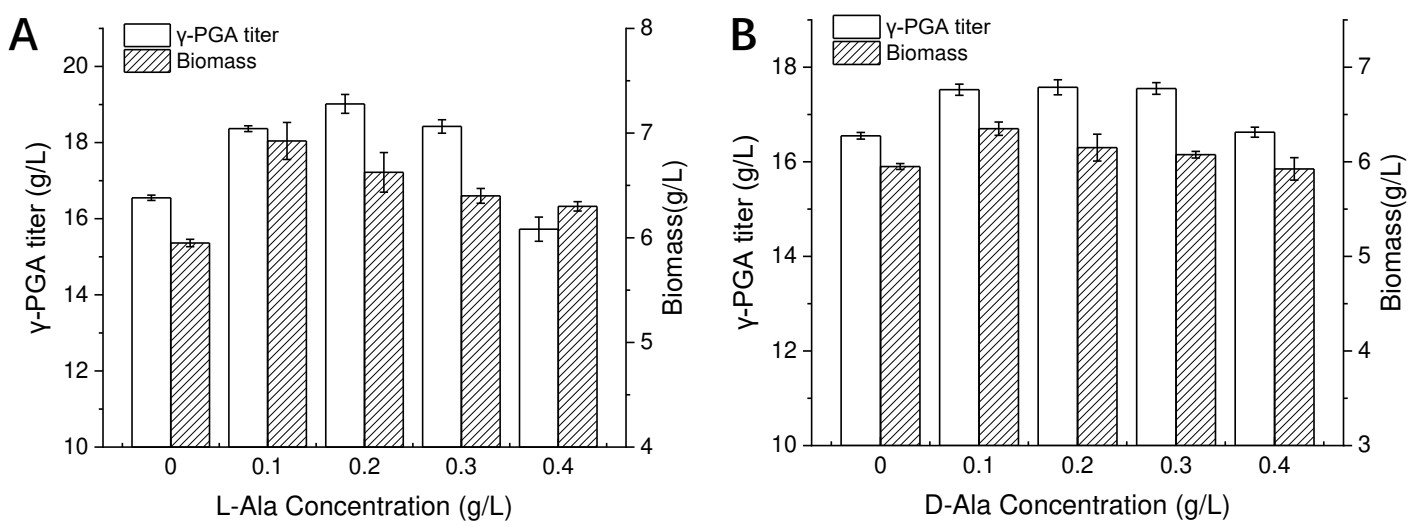

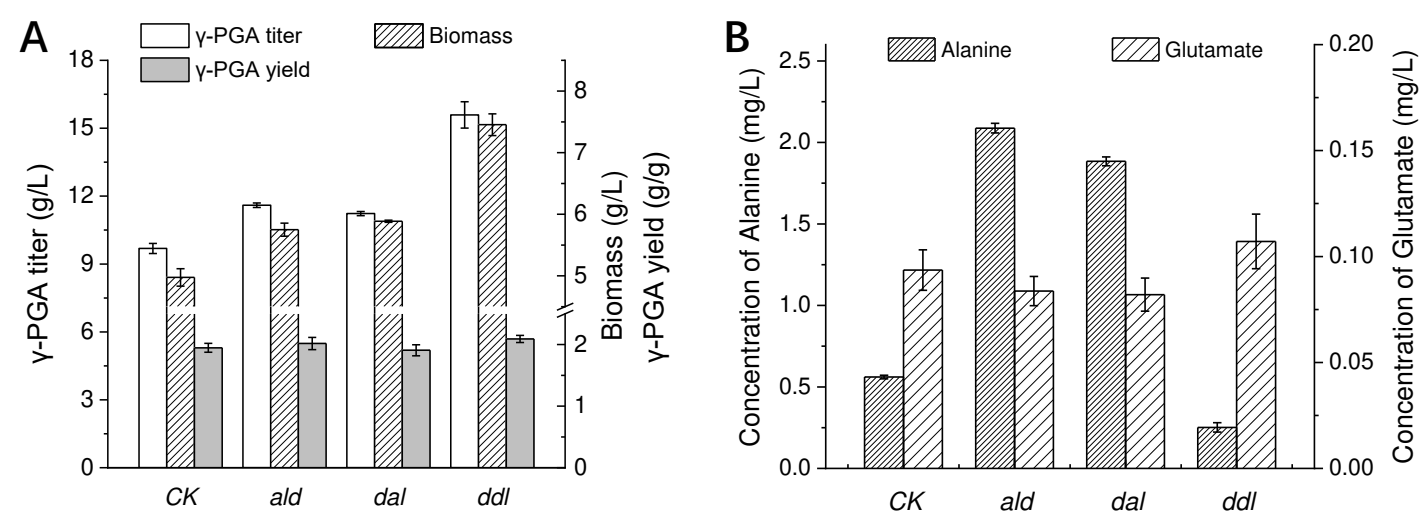

264 Fig. 4 Effects of genes ald, $d a l$ and $d d l$ overexpression on the production of $\gamma$-PGA (A) and the and glutamic acid were detected in mid-log phase. 